\title{
Peculiarities of THz-electromagnetic wave transmission through the GaN films under conditions of cyclotron and optical phonon transit-time resonances
}

\author{
V.V. Korotyeyev \\ V. Lashkaryov Institute of Semiconductor Physics, NAS of Ukraine, Department of Theoretical Physics \\ 41, prospect Nauky,03028Kyiv, Ukraine; e-mail: koroteev@ukr.net
}

\begin{abstract}
The theory of $\mathrm{THz}$ radiation transmission through the film of compensated $\mathrm{GaN}$ of cubic modification under action of electric and magnetic fields has been developed. In the $\mathrm{THz}$ frequency range, spectra of the dynamic mobility tensor have been obtained for applied steady-state electric fields of several $\mathrm{kV} / \mathrm{cm}$ and magnetic fields of several T. The spectra of transmission, reflection and loss coefficients have been analyzed for the Voigt configuration used for the applied fields. It has been shown that the transmitted wave becomes elliptically polarized, and the spectra of polarization characteristics such as ellipticity and rotation angle of the large axis of polarization ellipse were investigated under conditions of cyclotron resonance and optical phonon transit-time one. It has been found that, in certain frequency bands, polarization characteristics have the features valid for both resonances. The new optical method based on the analysis of ellipticity and rotation angle spectra is offered for determination of the low-field mobility.
\end{abstract}

Keywords: cyclotron resonance, optical phonon transit-time resonance.

Manuscript received 03.10.12; revised version received 20.11.12; accepted for publication 26.01.13; published online 28.02.13.

\section{Introduction}

In recent years, nitride materials, in particular GaN bulk samples and heterostructures, have attracted much attention due to perspectives of high-power and highfrequency applications. In spite numerous experimental and theoretical works devoted to transport properties of nitrides, studies of cyclotron resonance (CR) in these materials received much less attention. Under applied high magnetic field $\vec{H}$ the phenomenon of CR manifests itself in strong absorption of electromagnetic waves with the frequencies $\omega$ close to the cyclotron one, $\omega_{c}=e H / m^{*} c$, where $m^{*}$ is the electron effective mass, $e$ and $c$ are the elementary charge and light velocity, respectively. $\mathrm{CR}$ is one of powerful tools to characterize the basic material parameters. In particular, it is used for determinating the effective mass $\mathrm{m}^{*}$, scattering time $\tau$ and, consequently, mobility $\mu$. To observe wellresolved CR lines, it requires the condition $\omega_{c} \tau>1$. The CR experiments, dated by 90 s, were conducted basically for $\mathrm{AlGaN} / \mathrm{GaN}$ quantum heterostrucutures [1-3], while only one work was reported for GaN film [4]. In these works, the experiments were carried out at helium temperatures and even lower, and the values of electron mobility of the investigated samples were of a few thousands $\mathrm{cm}^{2} /(\mathrm{V} \cdot \mathrm{s})$. Typically, well-pronounced CR absorption was observed for the far-infrared radiation at $H=13 \mathrm{~T}$ in ref.[1] up to $40 \mathrm{~T}$ in ref.[3]. Weaklypronounced CR resonance was reported in refs. $[4,5]$ for the terahertz $(\mathrm{THz})$ radiation at moderate magnetic fields of several $\mathrm{T}$. The recent progress in the growth technique for high-quality nitride heterostructures with the electron mobility higher than several tens of thousands $\mathrm{cm}^{2} /(\mathrm{V} \cdot \mathrm{s})$ allowed to detect and study $\mathrm{CR}$ for the microwave 
radiation at the magnetic fields less than $1 \mathrm{~T}[6,7]$. Very often, in the cases of imperfect samples, the interpretation of the transmission spectra under CR conditions and determination of the electron mobility are difficult due to the large broadening of the CR absorption lines.

Here, we will show that for these cases the analysis of polarization characteristics of the transmitted wave can be more informative than those of transmission spectra. Furthermore, we will consider the peculiarities of the electromagnetic wave transmitted through the GaN film subjected to action of both magnetic fields and sufficiently strong steady-state (dc) electric fields. It is necessary to mention another resonance-like effect that can be met when studying the high-field electrical properties of nitrides. It is the effect of optical phonon transit-time resonance (OPTTR) which is associated with formation of a strongly anisotropic electron distribution function elongated along the applied dc electric field. This distribution function is the result of the cyclic, quasiballistic motion of electrons in momentum space interrupted by the fast emission of optical phonons. Under OPTTR conditions, frequency dispersion of dynamic (ac) conductivity has specific oscillating behavior with frequency intervals where the real part of conductivity becomes negative. For the GaN bulk-like samples $[8,9]$ and nitride quantum heterostructures $[10,11]$, OPTTR was predicted in the range of the applied dc electric fields of $1-10 \mathrm{kV} / \mathrm{cm}$ and a wide frequency range of about $0.3-2 \mathrm{THz}$. In spite of significant theoretical progress in description of OPTTR resonance, the experimental efforts aimed at measuring the transmission spectra [12] have not yet provided reliable evidences of OPTTR. However, the specific behavior of the polarization characteristics of transmitted wave can clearly indicate the existence of OPTTR.

\section{Formalism}

In this section, we present the theory of interaction of electromagnetic waves with a film of crystal of cubic modification with thickness $d$. We imply that $d$ is much greater than de Broglie wavelength of electrons, and, consequently, electron transport in the film can be considered as three-dimensional. Other dimensions of the film are assumed to be much greater than both $d$ and wavelength of the incident radiation. All the calculations will be provided assuming the parabolic dispersion law of electrons and the Voigt configuration of the applied fields (see Fig. 1). As shown in Fig. 1, the plane wave is incident upon the normal to plane of the film. The wavevector $\vec{k}$ of this wave is perpendicular to the applied steady-state fields $\vec{E}$ and $\vec{H}$, which are collinear and directed along $z$ axis. The electric vector $\vec{E}_{\omega}^{i n}$ of the incident wave lies in the plane $y=0$ and subtends the angle $\alpha$ with $z$ axis.

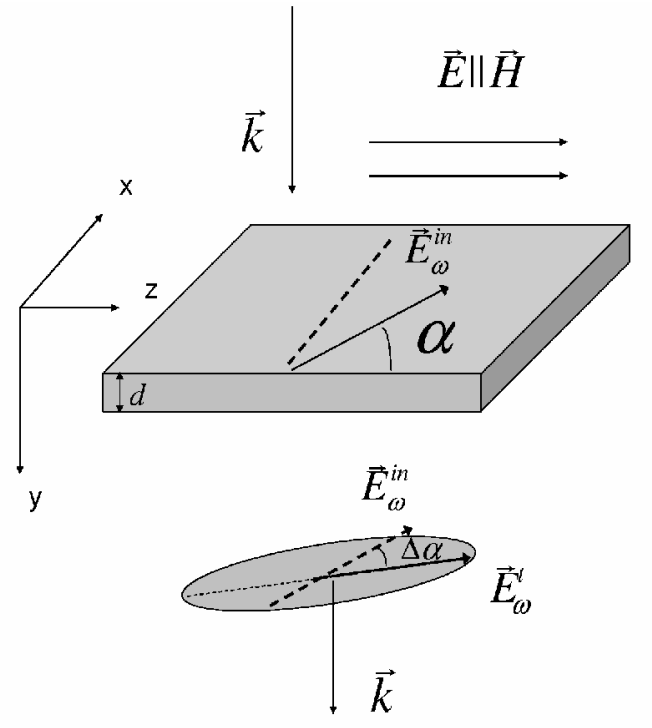

Fig. 1. Voigt configuration.

\section{a) Transmission, reflection and loss coefficients}

In the three considered regions: on, inside and under the film, the vector wave equation

$\operatorname{rot}\left(\operatorname{rot} \vec{E}_{\omega}\right)=-\frac{4 \pi}{c^{2}} \frac{\partial j_{\omega}}{\partial t}-\frac{\varepsilon}{c^{2}} \frac{\partial^{2} \vec{E}_{\omega}}{\partial t^{2}}$,

for the Voigt configuration can be written in the form of scalar equations as follows:

$\frac{d^{2} E_{\omega, i}}{d y^{2}}+ \begin{cases}\frac{\omega^{2}}{c^{2}} E_{\omega, i}, & y \leq 0 \\ \left(\frac{4 \pi I \omega \sigma_{i i}}{c^{2}}+\frac{\varepsilon \omega^{2}}{c^{2}}\right) E_{\omega, i}, & 0 \leq y \leq d \\ \frac{\omega^{2}}{c^{2}} E_{\omega, i}, & y \geq d\end{cases}$

where $I$ stands for imaginary unit; index $i=x, z ; \sigma_{i i}$ denotes diagonal components of the ac conductivity tensor; scalar quantity $\varepsilon$ is the background dielectric permittivity. The ac conductivity tensor is associated with the ac mobility tensor $\mu_{\omega}$ by the following relationship, $\sigma_{i j}=e n \mu_{\omega, i j}$, with $n$ denoting the electron concentration.

Generally speaking, in the presence of both strong dc electric and magnetic fields the dynamic or ac mobility, being the characteristic of the response of electron system to a small-signal ac perturbation, becomes the $3 \times 3$ tensor that for the assumed geometry of applied fields is written as

$\mu_{\omega}=\left(\begin{array}{ccc}\mu_{\omega, x x} & \mu_{\omega, x y} & 0 \\ \mu_{\omega, y x} & \mu_{\omega, y y} & 0 \\ 0 & 0 & \mu_{\omega, z z}\end{array}\right)$. 
In the case of the parabolic dispersion law, the dynamic mobility tensor contains only five non-zero components and only three of them $\mu_{\omega, x x}, \mu_{\omega, x y}$ and $\mu_{\omega, z z}$ are linearly independent. Other components are expressed as $\mu_{\omega, x x}=\mu_{\omega, y y}$ and $\mu_{\omega, x y}=-\mu_{\omega, y x}$. Note that at $H=0$ non-diagonal components are equal to zero. Being based on the parabolic dispersion law, the $\mu_{\omega, z z}$ component does not depend on $H$ and depends on the amplitudes of the electric field $\vec{E}$ and intensity of the electron scattering processes.

The system (2) has the following solutions:

$$
E_{\omega, i}= \begin{cases}A_{\omega, i} \exp \left(I k_{0} y\right)+B_{\omega, i} \exp \left(-I k_{0} y\right) & y \leq 0 \\ C_{\omega, i} \exp \left(I \lambda_{i} y\right)+D_{\omega, i} \exp \left(-I \lambda_{i} y\right) & 0 \leq y \leq d \\ E_{\omega, i}^{t} \exp \left(I k_{0} y\right) & y \geq d\end{cases}
$$

where $k_{0}=\omega / c$ and $\lambda_{i}=k_{0} \sqrt{\varepsilon} \sqrt{1+4 \pi \operatorname{Ien} \mu_{\omega, i i} / \omega \varepsilon}$ are the wave numbers in vacuum and in the film, respectively. Using the continuity conditions for the electric field $E_{\omega, i}$ and its derivative $d E_{\omega, i} / d y$ on both interfaces of the film, $y=0$ and $y=d$, we can express amplitudes of the $i$-components for the transmitted wave $E_{\omega, i}^{t}$ and reflected wave $B_{\omega, i}$ in the term of the amplitude of the incident wave $E_{\omega}^{i n}$. After some transformations, we obtain that $E_{\omega, i}^{t}=A_{\omega, i} t_{i} \exp \left(-I k_{0} d\right)$ and $\quad B_{\omega, i}=A_{\omega, i} r_{i} \quad$ (as can be seen in Fig. 1 $\left.A_{\omega, x, z}=E_{\omega}^{\text {in }}\{\sin (\alpha), \cos (\alpha)\}\right)$ where

$t_{i}=\left(\cos \left(\lambda_{i} d\right)-I \frac{\lambda_{i}^{2}+k_{0}^{2}}{2 \lambda_{i} k_{0}} \sin \left(\lambda_{i} d\right)\right)^{-1}$,

$r_{i}=I \frac{\lambda_{i}^{2}-k_{0}^{2}}{2 \lambda_{i} k_{0}} \sin \left(\lambda_{i} d\right) t_{i}$

Finally, the spectra of transmission, reflection and loss coefficients are given by the following formulas:

$T=\left|t_{x}\right|^{2} \sin ^{2}(\alpha)+\left|t_{z}\right|^{2} \cos ^{2}(\alpha)$

$R=\left|r_{x}\right|^{2} \sin ^{2}(\alpha)+\left|r_{z}\right|^{2} \cos ^{2}(\alpha) ; \quad L=1-T-R$.

As follows from Eqs. (7), at $\alpha=0^{\circ}$ (the incident wave polarized along $z$ axis), the spectra of transmission, reflection and loss coefficients will be defined only by the $\mu_{\omega, z z}$ component, and at $\alpha=90^{\circ}$ (the incident wave polarized along $x$ axis), the spectra of these coefficients will be defined only by $\mu_{\omega, x x}$. It is useful to note that at $\alpha \neq 0^{\circ}, 90^{\circ}$ the Voigt configuration allows to study both CR and OPTTR simultaneously, since CR manifests itself in the $\mu_{\omega, x x}$ component and OPTTR manifests itself in the $\mu_{\omega, z z}$ component.

\section{b) Polarization characteristics of the transmitted wave}

To clarify the physical properties of the transmitted wave, it is convenient to rewrite the components of its electric field in the real form:

$E_{\omega, x}^{t}(t)=E_{\omega}^{i n} \sin (\alpha)\left|t_{x}\right| \cos \left(\omega t-\varphi_{x}\right)$,

$E_{\omega, z}^{t}(t)=E_{\omega}^{i n} \cos (\alpha)\left|t_{z}\right| \cos \left(\omega t-\varphi_{z}\right)$,

where $\varphi_{i}=\arg \left(t_{i}\right)$. In the presence of $d c$ magnetic field $H$ or strong dc electric field $E$, the diagonal components $\mu_{\omega, i i}$ are different, therefore $\lambda_{x x} \neq \lambda_{z z}$ and, consequently, $\varphi_{x} \neq \varphi_{z}$. As a result, the transmitted wave at the incident angles $\alpha \neq 0^{\circ}, 90^{\circ}$ becomes elliptically polarized. In the $\{x, z\}$ plane, during one period of oscillation the electric vector will circumscribe some polarization ellipse. This ellipse can be characterized by ellipticity $\delta$ and rotation angle $\Delta \alpha$ of the large axis relatively to the initial angle $\alpha$. These quantities can be expressed as follows:

$\delta=\left[\frac{1-\sqrt{1-\beta^{2}}}{1-\sqrt{1+\beta^{2}}}\right]^{\frac{1}{2}}$

$\Delta \alpha=\arctan \left[\frac{2 p^{2}-\left(1+p^{2}\right)\left(1-\sqrt{1-\beta^{2}}\right)}{2 p \cos (\Delta \varphi)}\right]-\alpha$.

In the latter equations $p=\tan (\alpha)\left|t_{x} / t_{z}\right|$, $\beta=2 p \sin (\Delta \varphi) /\left(1+p^{2}\right) \quad$ and $\quad \Delta \varphi=\varphi_{x}-\varphi_{z} . \quad$ The essential simplification of the expressions (10) and (11) can be obtained in the limit of a thin film, when $\left|\lambda_{i} d\right|<<1$. To the first order of quantity $\lambda_{i} d$ smallness, the parameters of the polarization ellipse take the form convenient for analysis:

$\delta=\frac{\sin (2 \alpha)}{2} \frac{2 \pi d e n}{c}\left|\operatorname{Im}\left[\mu_{\omega, z z}\right]-\operatorname{Im}\left[\mu_{\omega, x x}\right]\right|$,
$\Delta \alpha=\frac{\sin (2 \alpha)}{2} \frac{2 \pi d e n}{c}\left[\operatorname{Re}\left[\mu_{\omega, z z}\right]-\operatorname{Re}\left[\mu_{\omega, x x}\right]\right]$.

As we can see, the spectra of $\delta$ and $\Delta \alpha$ are determined by the differences of imaginary and real parts of the $\mu_{\omega, z z}$ and $\mu_{\omega, x x}$ components of the dynamic mobility tensor, respectively. The maximal values of $\delta$ and $\Delta \alpha$ are realized at the incident angle $\alpha=45^{\circ}$. Also, it is useful to note that there are two characteristic frequencies $\omega_{1}$ and $\omega_{2}$ when $\delta=0$ and $\Delta \alpha=0$. They correspond to the intersections of the imaginary $\operatorname{Im}\left[\mu_{z z}\left(\omega_{1}\right)\right]=\operatorname{Im}\left[\mu_{\omega, x x}\left(\omega_{1}\right)\right] \quad$ and real $\operatorname{Re}\left[\mu_{z z}\left(\omega_{2}\right)\right]=\operatorname{Re}\left[\mu_{\omega, x x}\left(\omega_{2}\right)\right]$ parts of the diagonal components of the dynamic mobility tensor. The position of these two frequencies does not depend on the initial incident angle $\alpha$. As it will be shown below, determination of these characteristic frequencies could provide values of the scattering time. 


\section{Results and discussions}

We apply the theory developed in the section 2 to the film of compensated $\mathrm{GaN}$ of cubic modification with the following parameters: concentration of ionized impurity $N_{i}=10^{16} \mathrm{~cm}^{-3}$, electron concentration $n=10^{15} \mathrm{~cm}^{-3}$, ambient temperature $30 \mathrm{~K}$. The high-field and highfrequency properties of compensated bulk-like GaN have been analyzed in detail in ref.[13], and it was shown that at these parameters OPTTR is most pronounced within the range of the applied electric fields 2 to $8 \mathrm{kV} / \mathrm{cm}$. The low-field mobility $\mu$ was found to reach the values around $5000 \mathrm{~cm}^{2} /(\mathrm{V} \cdot \mathrm{s}$ ) (corresponding scattering time $\tau=0.57 \mathrm{ps}$ ). Note that the similar mobility values were measured experimentally in the free-standing $\mathrm{GaN}$ films [14].

Also, it is assumed the case of classical applied magnetic fields. The classical treatment of the magnetic field is satisfied when $\hbar \omega_{c}<\bar{\varepsilon}$, where $\bar{\varepsilon}$ is the average electron energy. In the equilibrium conditions, for example, at $\bar{\varepsilon} \approx 30 \mathrm{~K}$, the estimations give that the latter inequality is accomplished at $H<4.5 \mathrm{~T}$, which corresponds to $\omega_{c}<4 \mathrm{THz}$. In the highly nonequilibrium conditions, when the external dc electric field is applied with the amplitudes of several $\mathrm{kV} / \mathrm{cm}$, the average energy can reach essentially greater values of about $300 \mathrm{~K}$. Then, the range of the classical magnetic fields extends up to approximately $45 \mathrm{~T}$.

Below, these three different cases will be examined separately: the case of only $\mathrm{CR}(E=0, H \neq 0)$, the case of only OPTTR $(E \neq 0, H=0)$, and the case of the simultaneous coexistence of both $\mathrm{CR}$ and OPTTR $(E \neq 0, H \neq 0)$.

\section{a) The case of cyclotron resonance ( $E=0, H \neq 0$ )}

When external dc electric field is absent, the response of electron system to ac perturbation is described in the frames of the simple Drude-Lorentz model:

$$
\begin{aligned}
& \mu_{\omega, x x}=\mu \frac{1-I \omega \tau}{(1-I \omega \tau)^{2}+\left(\omega_{c} \tau\right)^{2}}, \\
& \mu_{\omega, z z}=\mu \frac{1}{1-\omega \tau},
\end{aligned}
$$

where time $\tau$ has a sense of the momentum relaxation time and relates to the low-field mobility as following: $\mu=e \tau / m^{*}$. Fig. 2a illustrates the frequency dependences of the components $\mu_{\omega, x x}$ and $\mu_{\omega, z z}$ calculated at $H=4.5 \mathrm{~T}$. As a result, at the magnetic field $\omega_{c} \tau=2.27$, the real part $\operatorname{Re}\left[\mu_{\omega, x x}\right]$ has a weak and quite a broadened peak corresponding to $\mathrm{CR}$. It is clearly reflected in the spectra of transmission and loss coefficients (curves 2 and 3 in the panels $(b)$ and $(d)$ ) and to a lesser degree in the spectrum of reflection coefficients (the same curves in the panel $(c)$ ). At the parameters assumed above for the GaN film, the maximal electromagnetic wave absorption occurs at polarization $\alpha=90^{\circ}$ and at the frequency $\omega / 2 \pi \approx \omega_{c} / 2 \pi=0.64 \mathrm{THz}$. The electromagnetic wave dissipates around $11 \%$ of its energy. At the same time, for other polarization $\alpha=0^{\circ}$, the electromagnetic wave dissipates around $3.5 \%$ of its energy. The transmission coefficient, which is directly measured quantity, is weakly changed from 79 to $71 \%$ with variation of $\alpha$ from $0^{\circ}$ to $90^{\circ}$. The accurate measurements of this small variation of transmission coefficients in $\mathrm{THz}$ frequency range can certainly face with serious experimental difficulties. In principle, it is general properties of thin samples with respect to wavelength of incident radiation and low electron concentration. As seen from Figs $2 b$ and $2 c$, electron gas with the concentration $n=10^{15} \mathrm{~cm}^{-3}$ weakly modifies frequency dispersion of the $T$ and $R$ coefficients (dash-dotted lines) of the ideal dielectric film with $n=0 \mathrm{~cm}^{-3}$. This fact can be illustrated by the estimations of the dimensionless parameters $\lambda_{x} d$ and $\lambda_{z} d$ : so at $\omega / 2 \pi=0.65 \mathrm{THz}, \quad \mu_{\omega, x x}=2600+I \times 520 \mathrm{~cm}^{2} /(\mathrm{V} \cdot \mathrm{s})$ and $\mu_{\omega, z z}=810+I \times 1840 \mathrm{~cm}^{2} /(\mathrm{V} \cdot \mathrm{s})$, which gives $\lambda_{x} d=0.393+I \times 0.027$ and $\lambda_{z} d=0.378+I \times 0.009$ at $d=10 \mu \mathrm{m}$. For comparison, $k_{0} \sqrt{\varepsilon} d=0.397$. Thus, the transmission and reflection coefficients of $\mathrm{THz}$ radiation are mainly conditioned by dielectric properties and geometry of the GaN film and are less affected by the electron subsystem.

However, as follows from formulas (12) and (13) the polarization characteristics $\delta$ and $\Delta \alpha$ are mainly conditioned by the electron subsystem. The spectra of these quantities are shown in Figs $3 b$ and $3 c$. It should be noted at once that the spectra of both $\delta$ and $\Delta \alpha$ calculated using the exact formulas (10) and (11) (solid lines) are close to the results obtained in the limit of thin film according to the formulas (12) and (13) (dashdotted lines). This is in the agreement with the estimations of the parameters $\lambda_{i} d$. One can see that the spectrum of ellipticity has highly non-monotonic behavior. There are two asymmetric "shoulders" separated by the united point (this point is fixed at every angle $\alpha$ ) where $\delta=0$. The frequency corresponding to this point is close to the characteristic frequency $\omega_{1}$ (right vertical line). The zero value of ellipticity means that the transmitted wave is linear polarized, and there is no phase shift between oscillations of the $x$ - and $z$ components of the electric field vector. The amplitude of the left "shoulder" of $\delta$ is much higher than that of the right "shoulder". It corresponds to larger difference, $\operatorname{Im}\left[\mu_{z z}(\omega)\right]-\operatorname{Im}\left[\mu_{\omega, x x}(\omega)\right]$ at $\omega<\omega_{1}$ than at $\omega>\omega_{1}$. The spectrum of the rotation angle also has the united point that separates the region of positive and negative values. The frequency corresponding to this point is 
close to the characteristic frequency $\omega_{2}$ (left vertical line). At frequencies $\quad \omega<\omega_{2}$ $\left(\operatorname{Re}\left[\mu_{z z}(\omega)\right]>\operatorname{Re}\left[\mu_{\omega, x x}(\omega)\right]\right)$, the electromagnetic wave transmitting through the sample has stronger extinction of the $z$-component of the electric field vector than $x$ component. As a result, the rotation angle is positive. At frequencies $\omega>\omega_{2}, \quad\left(\operatorname{Re}\left[\mu_{z z}(\omega)\right]<\operatorname{Re}\left[\mu_{\omega, x x}(\omega)\right]\right)$ and visa versa, as a result, the rotation angle is negative. In the high-frequency range where $\omega \tau>>\omega_{c} \tau>1$, the electron response becomes weaker, and electromagnetic wave transmitting through the samples remains practically linear polarized. For the parameters of the $\mathrm{GaN}$ film under consideration, the maximal values of ellipticity are of the order of several percents, and the rotation angle is approximately several degrees. These values can be easily determined experimentally with high accuracy [15].

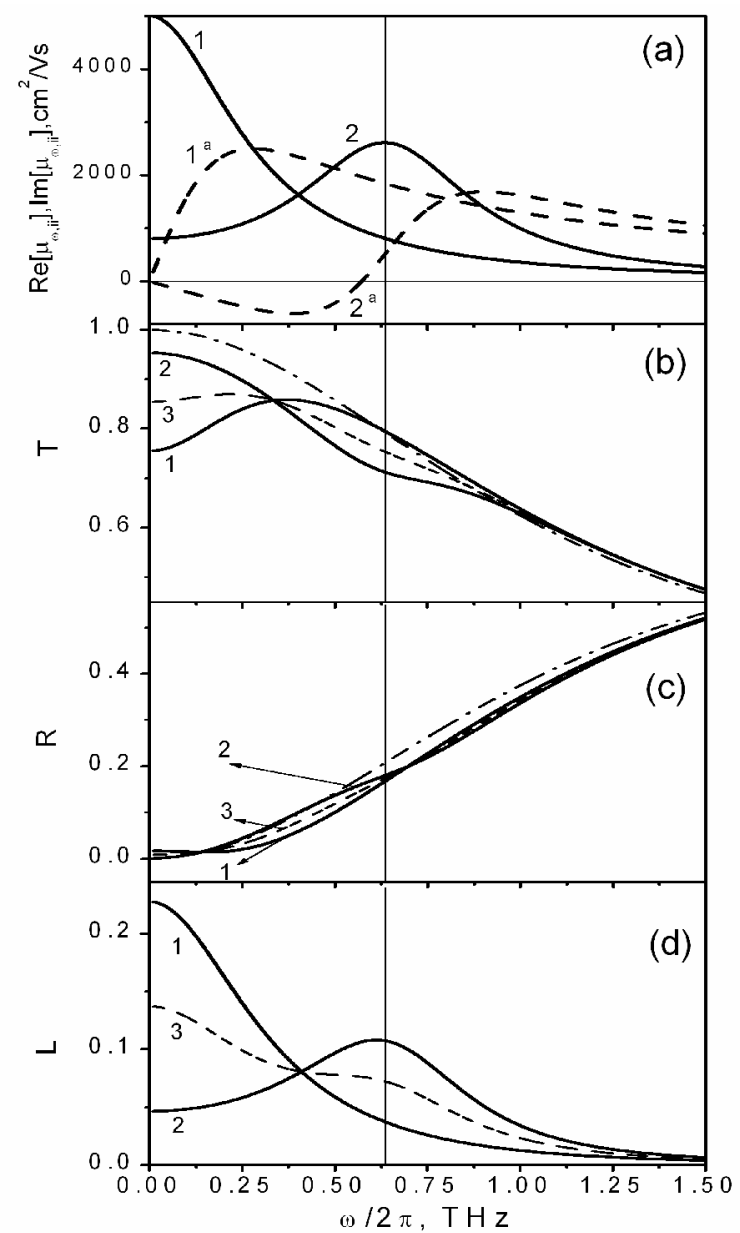

Fig. 2. Panel $(a)$ - spectra of $\mu_{\omega, z z}$ (curves 1 and $l^{a}$ are the real and imaginary parts, respectively) and $\mu_{\omega, x x}$ (curves 2 and $2^{a}$ ) calculated at $H=4.5 \mathrm{~T}\left(\omega_{c} / 2 \pi=0.64 \mathrm{THz}\right)$. Panels $(b),(c)$, $(d)$ - spectra of transmission, reflection and loss coefficients, respectively. Curves $1,2,3$ are obtained at $\alpha=0^{\circ}, 90^{\circ}, 45^{\circ}$, respectively. Dash-dotted lines are the results for pure dielectric film $n=0 \mathrm{~cm}^{-3}$. Vertical thin line denotes the position of cyclotron frequency $\omega_{c} / 2 \pi$. The thickness $d=10 \mu \mathrm{m}$. The dielectric permittivity $\varepsilon=8.9$.

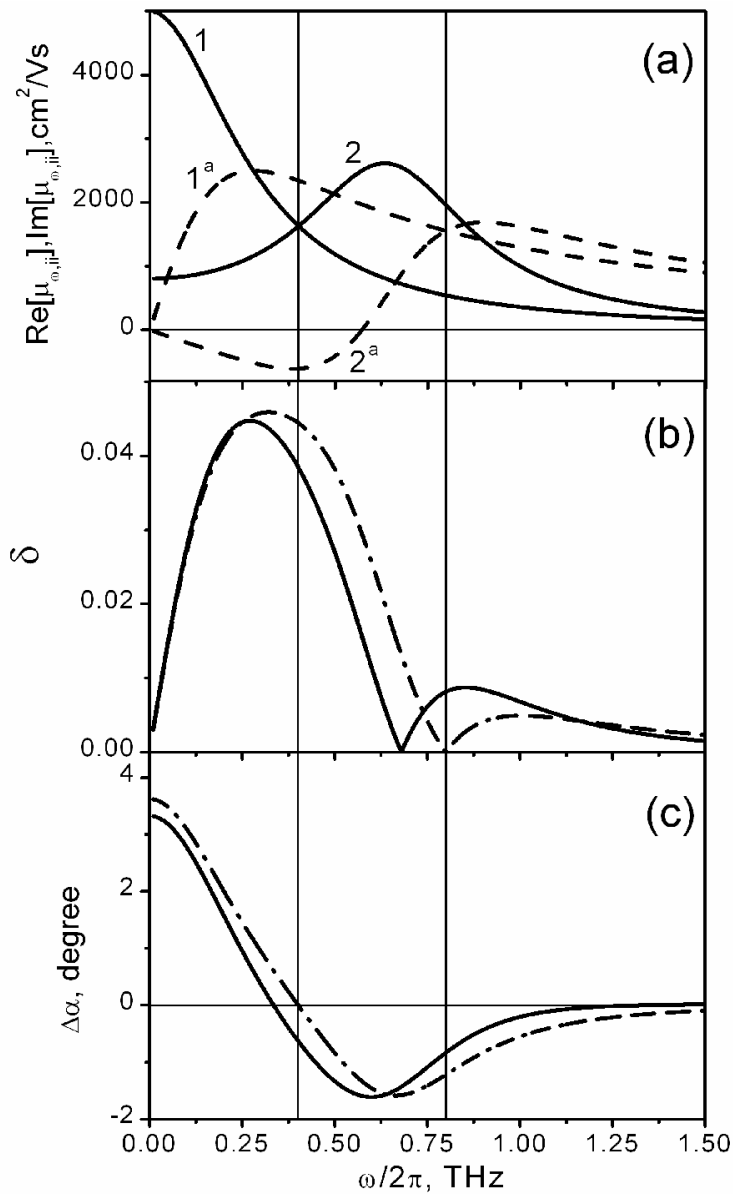

Fig. 3. Panel $(a)$ is the same as in Fig. 2. Panels $(b)$ and $(c)$ are the spectra of ellipticity and rotation angle, respectively. Solid lines are obtained according to the exact formulas (10) and (11), dash-dotted lines are obtained according to the approximate formulas (12) and (13). Vertical lines denote the position of characteristic frequency $\omega_{1} / 2 \pi$ and $\omega_{2} / 2 \pi$. The angle $\alpha=45^{\circ}$, other parameters are the same as in Fig. 2.

To finalize the discussion of $\mathrm{CR}$, it should be mentioned the possibility to determine the relaxation time by using the measurements of the polarization characteristics inherent to the transmitted wave. If the electron response obeys the Drude-Lorentz model (Eqs. (14) and (15)), then the characteristic frequencies $\omega_{1}$ and $\omega_{2}$ are explicitly defined as follows: $\omega_{1}^{2}=\omega_{c}^{2}+3 / \tau^{2} \quad$ and $\quad \omega_{2}^{2}=1 / 3 \times\left(\omega_{c}^{2}+1 / \tau^{2}\right)$. Experimentally determining the frequencies which correspond to $\delta=0$ or $\Delta \alpha=0$ (for the sufficiently thin films they practically coincide with $\omega_{1}$ and $\omega_{2}$ ) the relaxation time $\tau$ can be easily extracted using the latter formulas.

\section{b) The case of OPTT resonance ( $E \neq 0, H=0)$}

Under condition of the strong applied electric field the electron response cannot be described by the means of

\section{(C) 2013, V. Lashkaryov Institute of Semiconductor Physics, National Academy of Sciences of Ukraine}


the simple Drude-Lorentz model, and frequency dependences of mobility have to be found as based on the numerical solutions of Boltzman transport equation by using the Monte-Carlo technique. Analyzing the peculiarities of THz-light transmission, the spectra of dynamic mobility will be used from ref. [13]. The spectra of $\mu_{\omega, z z}$ and $\mu_{\omega, x x}$ under OPTTR are presented in Fig. 4a. As it is clear seen from Fig. 4a, the electron system has quite a different response to ac perturbation directed along and perpendicular to the dc electric field. This anisotropy of electron response is the result of formation of strongly anisotropic distribution function of electrons in the range of the sufficiently strong applied dc electric field. The component $\mu_{\omega, z z}$ reveals oscillating behavior with the frequency interval of $0.6-0.72 \mathrm{THz}$, where negative dynamic mobility (NDM) takes place. The maximum of NDM is realized near $0.65 \mathrm{THz}$ with

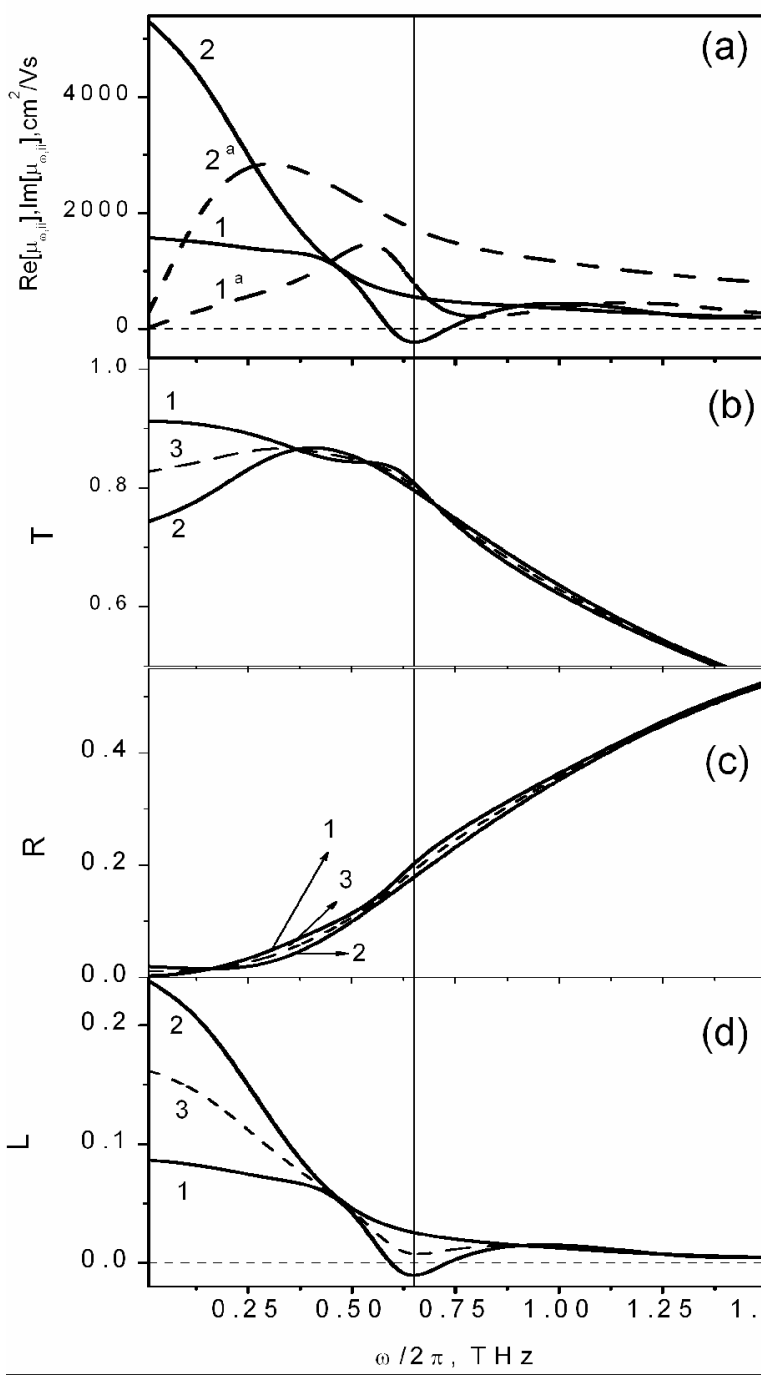

Fig. 4. Results are presented for $E=3 \mathrm{kV} / \mathrm{cm}$. All the curves have the same sense as in Fig. 2. the amplitudes around $225 \mathrm{~cm}^{2} /(\mathrm{V} \cdot \mathrm{s})$. At the same time, the component $\mu_{\omega, x x}$ does not reveal any visible features of OPTTR and remains Drude-like. In the frequency interval of OPTTR, the values of $\mu_{\omega, z z}$ component are by an order of magnitude smaller than those in the case of CR. Therefore, the signs of OPTTR in the transmission and reflection spectra (Figs $4 \mathrm{~b}$ and $4 \mathrm{c}$ ) are less pronounced than in the case of CR. In the frequency range of NDM, the loss coefficients at polarization $\alpha=0$ (curve 1 in Fig. 4 d) become negative, which indicates amplification of $\mathrm{THz}$ radiation. The intensity of transmitted wave is increased by $1 \%$ at the frequency $\omega / 2 \pi \sim 0.64 \mathrm{THz}$. Note, the amplification effect arising at OPTTR is sensitive to the direction of polarization of the incident wave. For example, at $\alpha>30^{\circ}$ the loss coefficient becomes positive and, instead of amplification, absorption of $\mathrm{THz}$ radiation takes place. At the same frequency but at polarization $\alpha=90^{\circ}$, the loss coefficient is equal to $2.5 \%$. The features of OPTTR in the spectra of $T, R, L$ coefficients appear to be more significant at a higher electron concentration. However, as shown in ref.[16], the effect of OPTTR is highly sensitive to the values of electron concentration and can be strongly suppressed in the dense electron gas due to electron-electron scattering.

In spite of formidable obstacles in identifying OPTTR resonance in the transmission spectra measurements, clear identification of OPTTR can be obtained by measuring the polarization characteristics of the transmitted wave. As seen from Figs $5 b$ and $5 c$, both spectra of ellipticity and rotation angle have oscillating behavior in the frequency range of OPTTR, which reflects oscillating behavior of the $\mu_{\omega, z z}$ component. However, in contrast to the previous case of $\mathrm{CR}$, the existence of united points corresponding to the frequencies when $\delta=0$ or $\Delta \alpha=0$ is not optional. For example, for the case under consideration, the ellipticity spectrum has no these points (note, the imaginary parts of $\mu_{\omega, z z}$ and $\mu_{\omega, x x}$ do not intersect), however, the rotation angle spectrum has three these points. Generally speaking, the existence of oscillation in the $\delta_{\omega}$ and $\Delta \alpha_{\omega}$ spectra is more representative for OPTTR than the existence of the united points. Also, in contrast to CR, in the low-frequency range $\operatorname{Re}\left[\mu_{\omega, z z}\right]<\operatorname{Re}\left[\mu_{\omega, x x}\right]$, and the rotation angle is negative. The amplitudes of $\Delta \alpha_{\omega}$ oscillation in the frequency range of OPTTR are about $1^{\circ}$, which can be also resolved experimentally.

\section{c) The case of the coexistence of $C R$ and OPTTR $(E \neq 0, H \neq 0)$}

The spectra of the $\mu_{\omega, z z}$ and $\mu_{\omega, x x}$ components of the dynamic mobility tensor obtained using the Monte-Carlo technique are presented in Fig. 6a for the case of parallel configuration of the applied dc electric and magnetic 


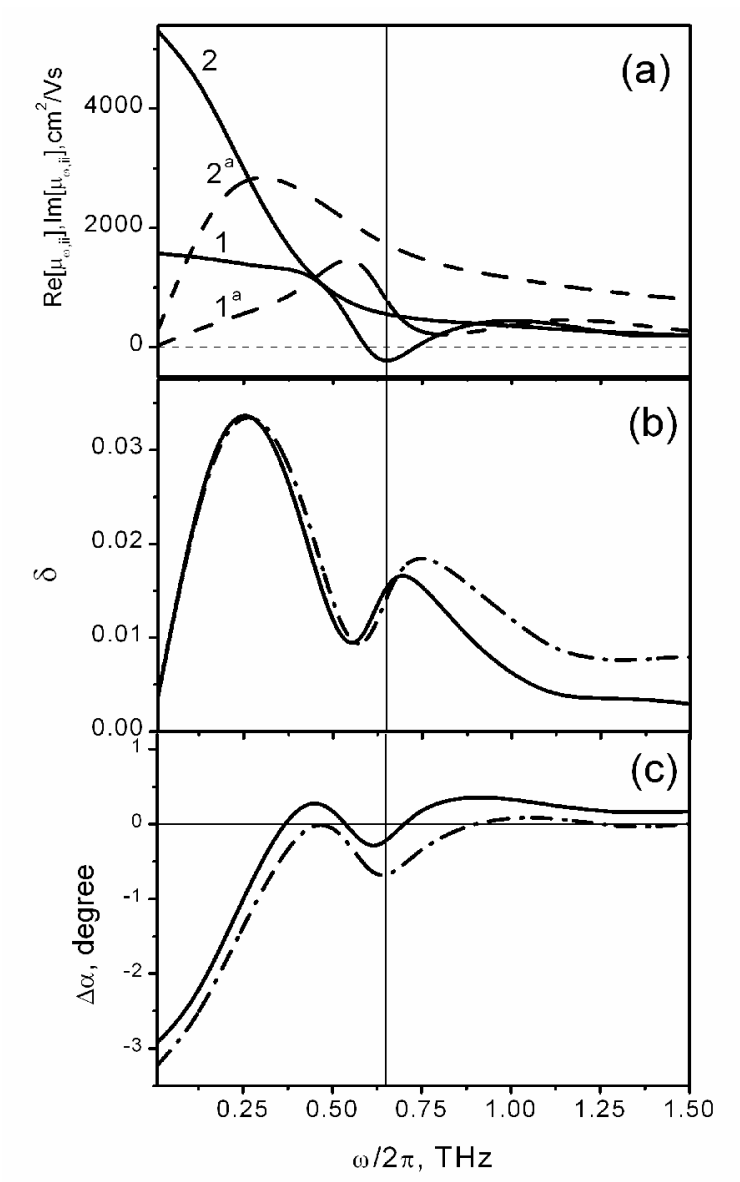

Fig. 5. Results are presented for $E=3 \mathrm{kV} / \mathrm{cm}$. All the curves have the same sense as in Fig. 3.

fields. In this configuration, it was established that the magnetic field does not change the electron response to ac perturbation directed along $z$ axes, and the $\mu_{\omega, z z}$ component remains the same as at $H=0$. This result is the consequence of the parabolic dispersion law of electrons, at which electron dynamics in magnetic field along $z$ axis and in the perpendicular directions are uncoupled. At the same time, the $\mu_{\omega, x x}$ component reveals specific behavior inherent to $\mathrm{CR}$. The shape of the spectrum of $\mu_{\omega, x x}$ component can be well-described by the Drude-Lorentz formula (14), introducing the same effective scattering time $\tau^{*}$. Under condition of OPTTR, this time corresponds to the average time of flight between two sequential processes of the optical phonon emission.

Thus, there is a possibility to study both effects simultaneously, analyzing transmission of $\mathrm{THz}$ radiation at different polarization angles $\alpha$. As seen from Figs $6 \mathrm{~b}$ and $6 \mathrm{~d}$, at the small incident angles $\alpha=0^{\circ}, 20^{\circ}$ (curves 1 and 3 ), the spectra of transmission and loss coefficients clearly demonstrate the sign of OPTTR: around the OPTTR frequency $\omega / 2 \pi=0.65 \mathrm{THz}$ there are a visible increase of the transmission coefficient and amplification of the transmitted wave (loss coefficient becomes negative and has the values $-0.3 \%$ down to $-0.1 \%$ ). The spectra of transmission and loss coefficients at the large angles of incidence $\alpha=75^{\circ}, 90^{\circ}$ (curves 5 and 2) instead of signs of OPTTR clearly demonstrate the signs of $\mathrm{CR}$. Around the $\mathrm{CR}$ frequency $\omega / 2 \pi=0.97 \mathrm{THz}$, there is a visible decrease of the transmission coefficient and absorption of the transmitted wave (loss coefficient becomes positive with the values close to $4 \%$ ). It is noteworthy that the absolute values of amplification and absorption are three times less than the values obtained previously in subsections (a) and (b). This is because the film thickness was chosen here three times less. At the angle $\alpha=45^{\circ}$ (curves 4 ), amplification is absent, but the $T$ and $L$ coefficients exhibit the signs inherent to both OPTTR and CR.

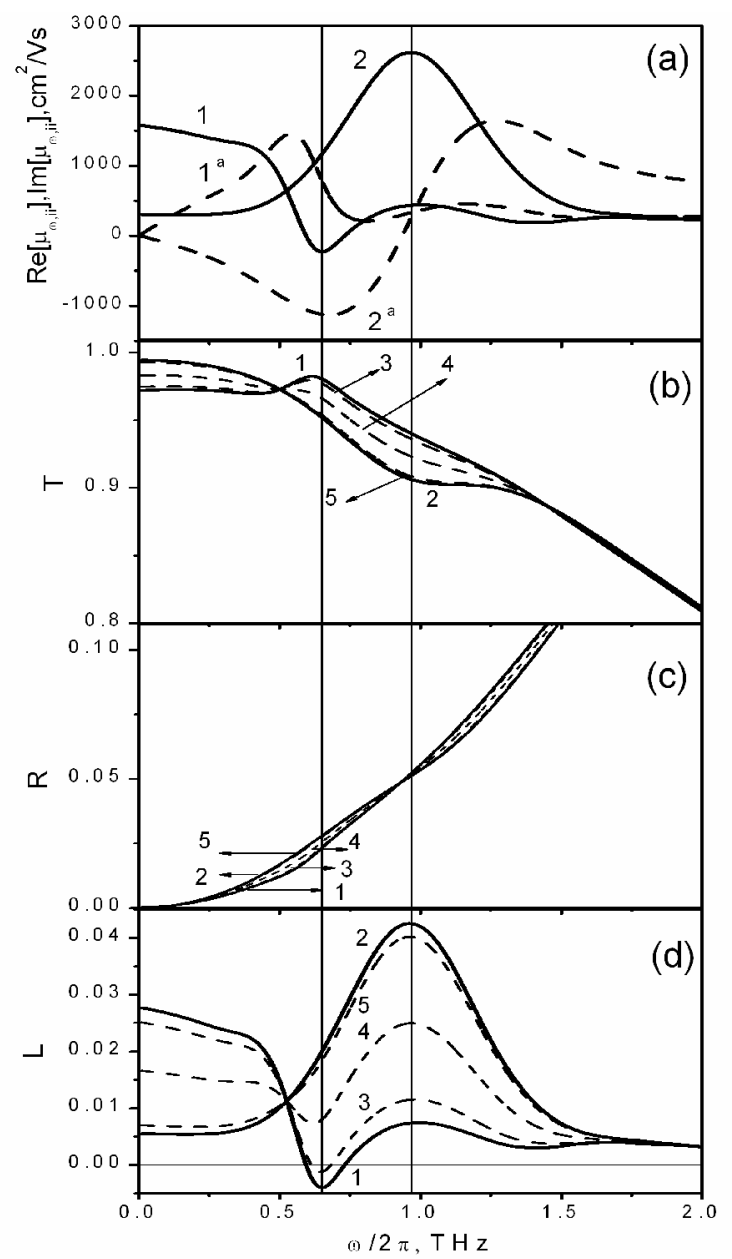

Fig. 6. Results are presented for $E=3 \mathrm{kV} / \mathrm{cm}$ and $H=6.8 \mathrm{~T}$. Vertical lines denote the position of the OPTTR frequency $\omega_{R} / 2 \pi=0.64 \mathrm{THz}$ and the CR frequency $\omega_{c} / 2 \pi=0.97 \mathrm{THz}$. The curves $3,4,5$ are obtained at $\alpha=20^{\circ}, 45^{\circ}, 75^{\circ}$. Other curves have the same sense as in Fig. 2. The thickness of the film $d=3 \mu \mathrm{m}$. 


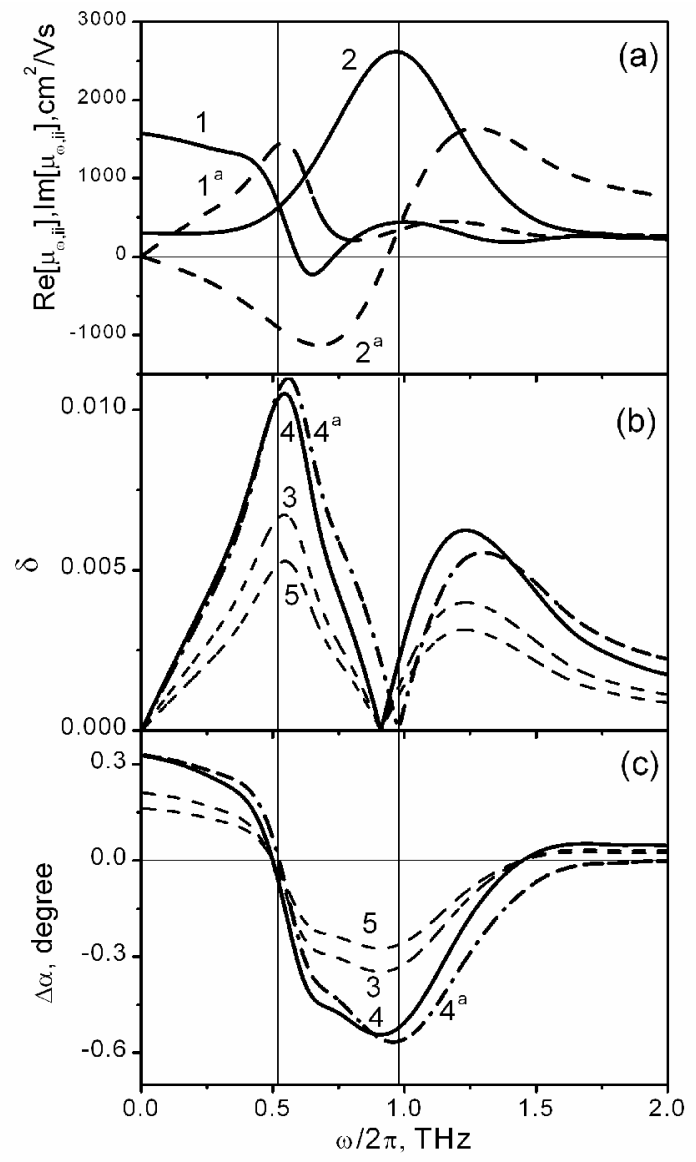

Fig. 7. Curves 3,4 , and 5 in panels $(b)$ and $(c)$ are obtained at $\alpha=20^{\circ}, 45^{\circ}, 75^{\circ}$. The dash-dotted curve presents the approximation of thin film and it is calculated at $\alpha=45^{\circ}$. Other parameters are the same as in Fig. 6.

The same simultaneous manifestation of these effects is observed in the frequency dependences of polarization characteristics $\delta$ and $\Delta \alpha$ (Figs 7b and 7c). There are united points corresponding to the frequencies $\delta=0$ and $\Delta \alpha=0$ typical for CR, and there is inconspicuous frequency modulation both $\delta$ and $\Delta \alpha$ typical for OPTTR.

\section{Conclusions}

In summary, the measurements of the polarization characteristics of transmitted wave through the sample are no less important than the measurements of the transmission spectra. In the cases of thin films, such polarization characteristics of radiation as ellipticity and rotation angle are mainly determined by the highfrequency conductivity of the electron subsystem. The widely discussed in literature effects of optical phonon transit-time resonance and cyclotron resonance can be experimentally identified by the distinctive features of the spectra of ellipticity as well as those of the rotation angle. In the case of cyclotron resonance, the investigations of polarization characteristics can enable to determine the electron relaxation time and, consequently, the low-field mobility.

\section{Acknowledgement}

This work has been carried out in the framework of the Complex Program of the Fundamental Researches of NAS of Ukraine "Fundamental problems of nanostructured systems, materials and nanotechnology" (2010-2014) and partially supported by The State Fund for Fundamental Researches (project $\Phi 40.2 / 057$ ).

\section{References}

1. W. Knap, H. Alause, J.M. Bluet, J. Camassel, J. Young, M. Asif Khan, Q. Chen, S. Huant, M. Shur, The cyclotron resonance effective mass of two-dimensional electrons confined at the GaN/AlGaN interface // Solid State Communs. 99(3), p. 195-199 (1996).

2. Xiaoguang $\mathrm{Wu}$ and F.M. Peeters, Cyclotronresonance mass of two-dimensional electrons in $\mathrm{GaN} / \mathrm{Al}_{\mathrm{x}} \mathrm{Ga}_{1-\mathrm{x}} \mathrm{N}$ heterostructures // Phys. Rev. B, 55(23), p. 15438-15440 (1997).

3. W. Knap, S. Contreras, H. Alause, C. Skierbiszewski, J. Camassel et al., Cyclotron resonance and quantum Hall effect studies of the two-dimensional electron gas confined at the GaN/AlGaN interface // Appl. Phys. Lett. 70, p. 2123-2125 (1997).

4. M. Drechsler, D.M. Hofmann, B.K. Meyer, T. Detchprohm, H. Amano and I. Akasaki, Determination of the conduction band electron effective mass in hexagonal GaN // Jpn. J. Appl. Phys. 34, p. L1178-L1179 (1995).

5. Z.-F. Li, W. Lu, S.C. Shen, S. Holland, C.M. Hu et al., Cyclotron resonance and magnetotransport measurements in $\mathrm{Al}_{\mathrm{x}} \mathrm{Ga}_{1-\mathrm{x}} \mathrm{N} / \mathrm{GaN}$ heterostructures for $\mathrm{x}=0.15-0.30 / / \mathrm{Appl}$. Phys. Lett. 80(3), p. 431-433(2002).

6. S. Syed, M.J. Manfra, Y.J. Wang, R.J. Molnar and H.L. Stormer, Electron scattering in $\mathrm{AlGaN} / \mathrm{GaN}$ structures // Appl. Phys. Lett. 84(9), p. 1507-1509 (2004).

7. A. Wolos, W. Jantsch, K. Dybko and Z. Wilamowski, C. Skierbiszewski, Plasmoncyclotron resonance in two-dimensional electron gas confined at the $\mathrm{GaN} / \mathrm{Al}_{\mathrm{x}} \mathrm{Ga}_{1-\mathrm{x}} \mathrm{N}$ interface // Phys. Rev. B, 76, 045301 (2007).

8. E. Starikov, P. Shiktorov, V. Gruzinskis, L. Reggiani, L. Varani, J.C. Vaissiere and Jian H. Zhao, Monte Carlo simulation of the generation of terahertz radiation in GaN // J. Appl. Phys. 89(2), p. 1161-1171 (2001); E.A. Barry, K.W. Kim, and V.A. Kochelap, Group-III nitrides hot electron effects in moderate electric fields // Phys. Status Solidi (B), 228(2), p. 571-574 (2001). 
9. G.I. Syngayivska and V.V. Korotyeyev, Monte Carlo simulation of hot electron effects in compensated GaN semiconductor at moderate electric fields // Semiconductor Physics, Quantum Electronics \& Optoelectronics, 10(4), p. 54-59 (2007).

10. V.V. Korotyeyev, V.A. Kochelap, K.W. Kim and D.L. Woolard, Streaming distribution of twodimensional electrons in III-N heterostructures for electrically pumped terahertz generation // Appl. Phys. Lett. 82, p. 2643-2645 (2003); K.W. Kim, V.V. Korotyeyev, V.A. Kochelap, A.A. Klimov, and D.L. Woolard, Tunable terahertz-frequency resonances and negative dynamic conductivity of two-dimensional electrons in group-III nitrides // $\mathrm{J}$. Appl. Phys. 96, p. 6488-6491 (2004).

11. E. Starikov, P. Shiktorov, V. Gruzinskis, L. Varani, C. Palermo, J.-F. Millithaler and L. Regiani, Frequency limits of terahertz radiation generated by optical-phonon transit-time resonance in quantum wells and heterolayers // Phys. Rev. B, 76, 045333 (2007); Terahertz generation in nitrides due to transittime resonance assisted by optical phonon emission // J. Phys: Condens. Matter, 20(38), 384209 (2008).
12. T. Laurent, R. Sharma, J. Torres, P. Nouvel, S. Blin, L. Varani, Y. Cordier, M. Chmielowska, S. Chenot, J.-P. Faurie, B. Beaumont, P. Shiktorov, E. Starikov, V. Gruzinskis, V.V. Korotyeyev, and V.A. Kochelap, Voltage-controlled sub-terahertz radiation transmission through GaN quantum well structure // Appl. Phys. Lett. 99, 082101 (2011).

13. G.I. Syngayivska, V.V. Korotyeyev, Electrical and high-frequency properties of compensated GaN under electron streaming conditions// Ukrainian Journal of Physics, 58 (1), p. 40-55 (2013).

14. D.C. Look and J.R. Sizelove, Predicted maximum mobility in bulk GaN // Appl. Phys. Lett. 79(8), p. 1133-1135 (2001).

15. L. Ren, C.L. Pint, T. Arikawa, K. Takeya, I. Kawayama, M. Tonouchi, R.H. Hauge, and J. Kono, Broadband terahertz polarizers with ideal performance based on aligned carbon nanotube stacks // Nano Lett., 12, p. 787-790 (2012).

16. J.T. Lu, J.C. Cao, and S.L. Feng, Hot-electron dynamics and terahertz generation in GaN quantum wells in the streaming transport regime // Phys. Rev. B, 73, 195326 (2006). 